\title{
Improving the Recognizability of Syntactic Relations Using Contextualized Examples
}

\author{
Aditi Muralidharan \\ Computer Science Division \\ University of California, Berkeley \\ Berkeley, CA \\ asmeberkeley .edu
}

\author{
Marti A. Hearst \\ School of Information \\ University of California, Berkeley \\ Berkeley, CA \\ hearst@berkeley .edu
}

\begin{abstract}
A common task in qualitative data analysis is to characterize the usage of a linguistic entity by issuing queries over syntactic relations between words. Previous interfaces for searching over syntactic structures require programming-style queries. User interface research suggests that it is easier to recognize a pattern than to compose it from scratch; therefore, interfaces for non-experts should show previews of syntactic relations. What these previews should look like is an open question that we explored with a 400-participant Mechanical Turk experiment. We found that syntactic relations are recognized with $34 \%$ higher accuracy when contextual examples are shown than a baseline of naming the relations alone. This suggests that user interfaces should display contextual examples of syntactic relations to help users choose between different relations.
\end{abstract}

\section{Introduction}

The ability to search over grammatical relationships between words is useful in many nonscientific fields. For example, a social scientist trying to characterize different perspectives on immigration might ask how adjectives applying to 'immigrant' have changed in the last 30 years. A scholar interested in gender might search a collection to find out whether different nouns enter into possessive relationships with 'his' and 'her' (Muralidharan and Hearst, 2013). In other fields, grammatical queries can be used to develop patterns for recognizing entities in text, such as medical terms (Hirschman et al., 2005; MacLean and Heer, 2013), and products and organizations $(\mathrm{Cu}-$ lotta and McCallum, 2005), and for coding qualitative data such as survey results.
Most existing interfaces for syntactic search (querying over grammatical and syntactic structures) require structured query syntax. For example, the popular Stanford Parser includes Tregex, which allows for sophisticated regular expression search over syntactic tree structures (Levy and Andrew, 2006). The Finite Structure Query tool for querying syntactically annotated corpora requires its queries to be stated in first order logic (Kepser, 2003). In the Corpus Query Language (Jakubicek et al., 2010), a query is a pattern of attributevalue pairs, where values can include regular expressions containing parse tree nodes and words. Several approaches have adopted XML representations and the associated query language families of XPATH and SPARQL. For example, LPath augments XPath with additional tree operators to give it further expressiveness (Lai and Bird, 2010).

However, most potential users do not have programming expertise, and are not likely to be at ease composing rigidly-structured queries. One survey found that even though linguists wished to make very technical linguistic queries, $55 \%$ of them did not know how to program (Soehn et al., 2008). In another (Gibbs and Owens, 2012), humanities scholars and social scientists are frequently skeptical of digital tools, because they are often difficult to use. This reduces the likelihood that existing structured-query tools for syntactic search will be usable by non-programmers (Ogden and Brooks, 1983).

A related approach is the query-by-example work seen in the past in interfaces to database systems (Androutsopoulos et al., 1995). For instance, the Linguist's Search Engine (Resnik et al., 2005) uses a query-by-example strategy in which a user types in an initial sentence in English, and the system produces a graphical view of a parse tree as output, which the user can alter. The user can either click on the tree or modify the LISP expression to generalize the query. SPLICR also contains 
a graphical tree editor tool (Rehm et al., 2009). According to Shneiderman and Plaisant (2010), query-by-example has largely fallen out of favor as a user interface design approach. A downside of QBE is that the user must manipulate an example to arrive at the desired generalization.

More recently auto-suggest, a faster technique that does not require the manipulation of query by example, has become a widely-used approach in search user interfaces with strong support in terms of its usability (Anick and Kantamneni, 2008; Ward et al., 2012; Jagadish et al., 2007). A list of selectable options is shown under the search bar, filtered to be relevant as the searcher types. Searchers can recognize and select the option that matches their information need, without having to generate the query themselves.

The success of auto-suggest depends upon showing users options they can recognize. However, we know of no prior work on how to display grammatical relations so that they can be easily recognized. One current presentation (not used with auto-suggest) is to name the relation and show blanks where the words that satisfy it would appear as in $X$ is the subject of $Y$ (Muralidharan and Hearst, 2013); we used this as the baseline presentation in our experiments because it employs the relation definitions found in the Stanford Dependency Parser's manual (De Marneffe et al., 2006). Following the principle of recognition over recall, we hypothesized that showing contextualized usage examples would make the relations more recognizable.

Our results confirm that showing examples in the form of words or phrases significantly improves the accuracy with which grammatical relationships are recognized over the standard baseline of showing the relation name with blanks. Our findings also showed that clausal relationships, which span longer distances in sentences, benefited significantly more from example phrases than either of the other treatments.

These findings suggest that a query interface in which a user enters a word of interest and the system shows candidate grammatical relations augmented with examples from the text will be more successful than the baseline of simply naming the relation and showing gaps where the participating words appear.

\section{Experiment}

We gave participants a series of identification tasks. In each task, they were shown a list of sentences containing a particular syntactic relationship between highlighted words. They were asked to identify the relationship type from a list of four options. We presented the options in three different ways, and compared the accuracy.

We chose Amazon's Mechanical Turk (MTurk) crowdsourcing platform as a source of study participants. The wide range of backgrounds provided by MTurk is desirable because our goal is to find a representation that is understandable to most people, not just linguistic experts or programmers. This platform has become widely used for both obtaining language judgements and for usability studies (Kittur et al., 2008; Snow et al., 2008).

Our hypothesis was:

Grammatical relations are identified more accurately when shown with examples of contextualizing words or phrases than without.

To test it, participants were given a series of identification tasks. In each task, they were shown a list of 8 sentences, each containing a particular relationship between highlighted words. They were asked to identify the relationship from a list of 4 choices. Additionally, one word was chosen as a focus word that was present in all the sentences, to make the relationship more recognizable ("life" in Figure 1).

The choices were displayed in 3 different ways (Figure 1). The baseline presentation (Figure 1a) named the linguistic relation and showed a blank space with a pink background for the varying word in the relationship, the focus word highlighted in yellow and underlined, and any necessary additional words necessary to convey the relationship (such as "of" for the prepositional relationship "of", the third option).

The words presentation showed the baseline design, and in addition beneath was the word "Examples:" followed by a list of 4 example words that could fill in the pink blank slot (Figure 1b). The phrases presentation again showed the baseline design, beneath which was the phrase "Patterns like:" and a list of 4 example phrases in which fragments of text including both the pink and the yellow highlighted portions of the relationship appeared (Figure 1c). 


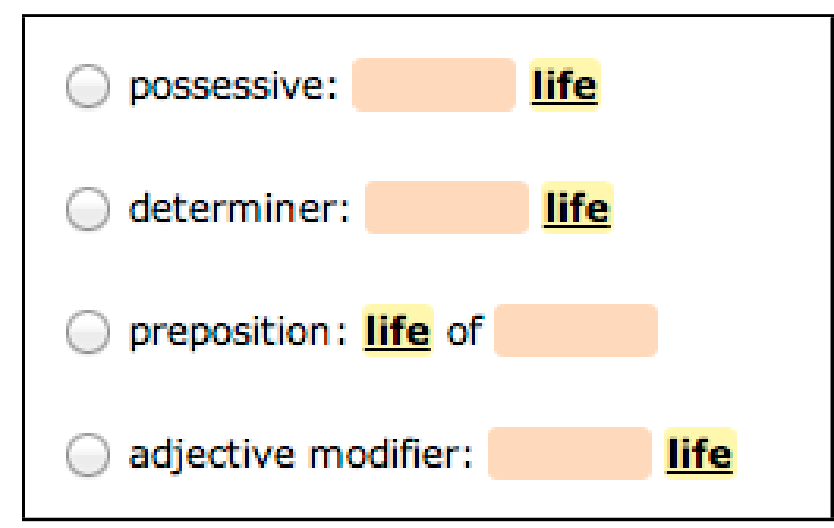

(a) The options as they appear in the baseline condition.

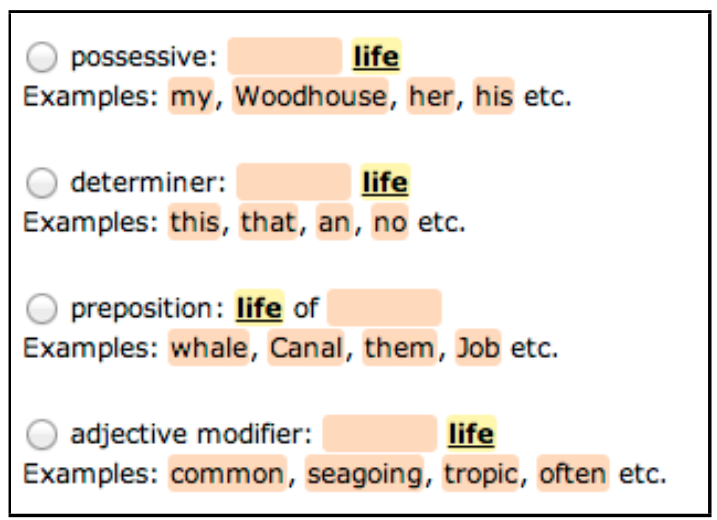

(b) The same options as they appear in the words condition.

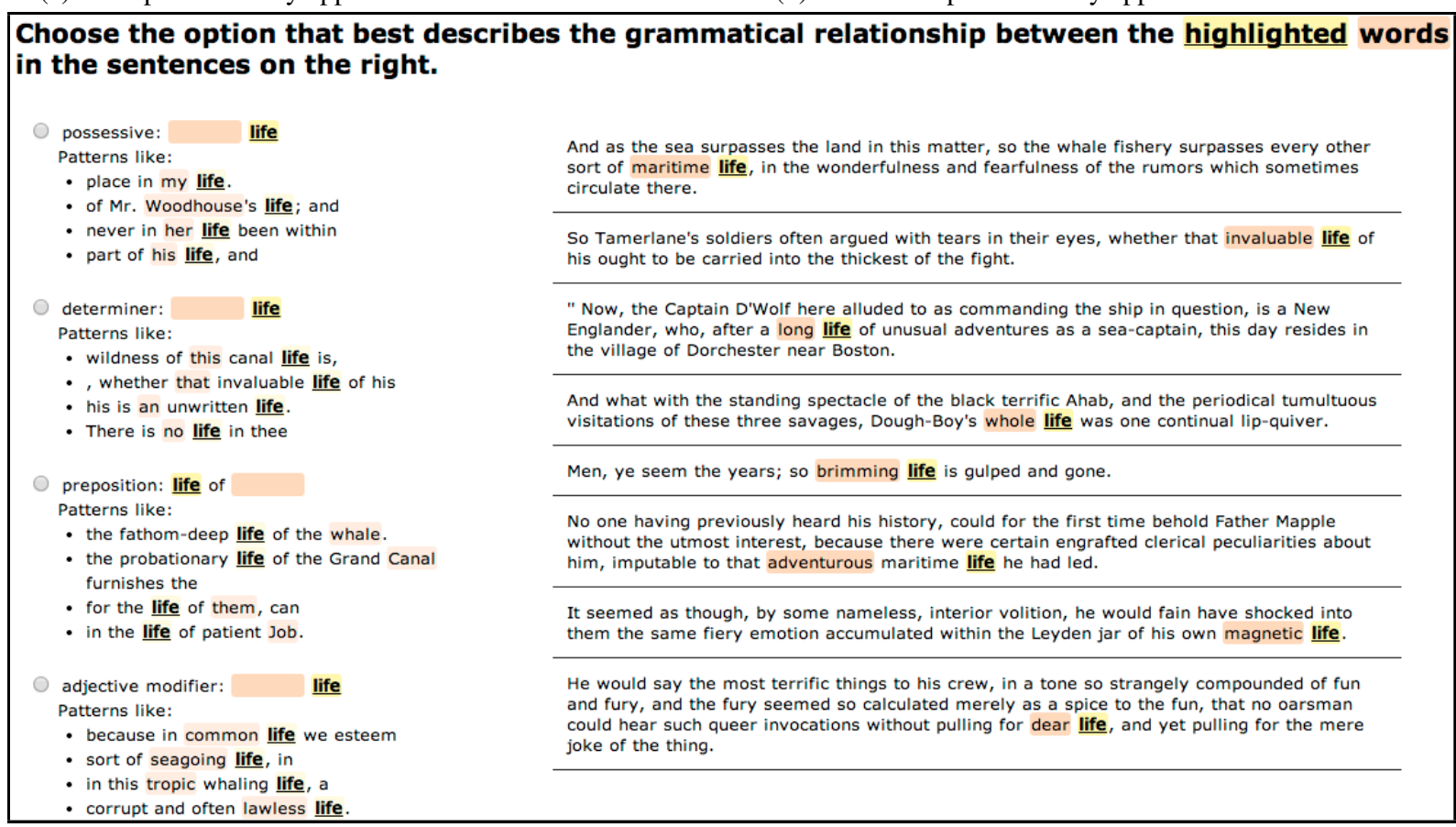

(c) The same options in the phrases condition, shown as they appeared in an identification task for the relationship amod ( life, _--) (where different adjectives modify the noun 'life'). The correct answer is 'adjective modifier' (4th option), and the remaining 3 options are distractors.

Figure 1: The appearance of the choices shown in the three experiment conditions.

Method: We used a between-subjects design. The task order and the choice order were not varied: the only variation between participants was the presentation of the choices. To avoid the possibility of guessing the right answer by patternmatching, we ensured that there was no overlap between the list of sentences shown, and the examples shown in the choices as words or phrases.

Tasks: The tasks were generated using the Stanford Dependency Parser (De Marneffe et al., 2006) on the text of Moby Dick by Herman Melville. We tested the 12 most common grammatical relationships in the novel in order to cover the most content and to be able to provide as many real examples as possible. These relationships fell into two categories, listed below with examples.

Clausal or long-distance relations:

- Adverbial clause: I walk while talking

- Open clausal complement: I love to sing

- Clausal complement: he saw us leave

- Relative clause modifier: the letter I wrote reached

Non-clausal relations:

- Subject of verb: he threw the ball

- Object of verb: he threw the ball

- Adjective modifier red ball

- Preposition (in): a hole in a bucket

- Preposition (of): the piece of cheese

- Conjunction (and) mind and body 
- Adverb modifier: we walk slowly

- Noun compound: Mr. Brown

We tested each of these 12 relations with 4 different focus words, 2 in each role. For example, the Subject of Verb relation was tested in the following forms:

- (Ahab, _-- ): the sentences each contained 'Ahab', highlighted in yellow, as the subject of different verbs highlighted in pink.

- (captain, ---)

- (_--, said): the sentences each contained the verb 'said', highlighted in yellow, but with different subjects, highlighted in pink.

- (,-- stood)

To maximize coverage, yet keep the total task time reasonable (average 6.8 minutes), we divided the relations above into 4 task sets, each testing recognition of 3 different relations. Each of relations was tested with 4 different words, making a total of 12 tasks per participant.

Participants: 400 participants completed the study distributed randomly over the 4 task sets and the 3 presentations. Participants were paid 50c (U.S.) for completing the study, with an additional $50 \mathrm{c}$ bonus if they correctly identified 10 or more of the 12 relationships. They were informed of the possibility of the bonus before starting.

To gauge their syntactic familiarity, we also asked them to rate how familiar they were with the terms 'adjective' ( $88 \%$ claimed they could define it), 'infinitive' (43\%), and 'clausal complement' $(18 \%)$. To help ensure the quality of effort, we included a multiple-choice screening question, "What is the third word of this sentence?" The 27 participants (out of 410) who answered incorrectly were eliminated.

Results: The results (Figure 2) confirm our hypothesis. Participants in conditions that showed examples (phrases and words) were significantly more accurate at identifying the relations than participants in the baseline condition. We used the Wilcoxson signed-rank test, an alternative to the standard T-test that does not assume samples are normally distributed. The average success rate in the baseline condition was $41 \%$, which is significantly less accurate than words: 52\%, $(\mathrm{p}=0.00019, \mathrm{~W}=6136)$, and phrases: $55 \%$, $(\mathrm{p}=0.00014, \mathrm{~W}=5546.5)$.

Clausal relations operate over longer distances in sentences, and so it is to be expected that showing longer stretches of context would perform bet-

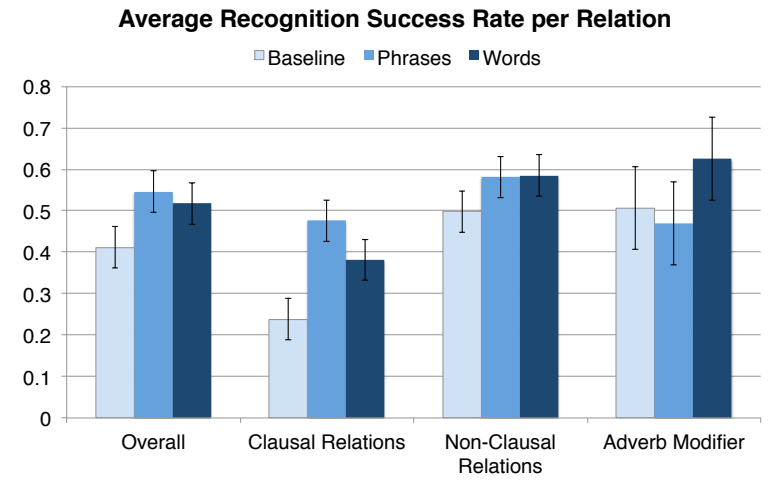

Figure 2: Recognition rates for different types of relations under the 3 experiment conditions, with $95 \%$ confidence intervals.

ter in these cases; that is indeed what the results showed. Phrases significantly outperformed words and baseline for clausal relations. The average success rate was $48 \%$ for phrases, which is significantly more than words: $38 \%,(\mathrm{p}=0.017$ $\mathrm{W}=6976.5)$ and baseline: $24 \%, \quad\left(\mathrm{p}=1.9 \times 10^{-9}\right.$ $\mathrm{W}=4399.0$ ), which was indistinguishable from random guessing $(25 \%)$. This is a strong improvement, given that only $18 \%$ of participants reported being able to define 'clausal complement'.

For the non-clausal relations, there was no significant difference between phrases and words, although they were both overall significantly better than the baseline (words: $\mathrm{p}=0.0063 \mathrm{~W}=6740$, phrases: $\mathrm{p}=0.023 \mathrm{~W}=6418.5$ ). Among these relations, adverb modifiers stood out (Figure 2), because evidence suggested that words $(63 \%$ success) made the relation more recognizable than phrases $(47 \%$ success, $\mathrm{p}=0.056, \mathrm{~W}=574.0)$ - but the difference was only almost significant, due to the smaller sample size (only 96 participants encountered this relation). This may be because the words are the most salient piece of information in an adverbial relation - adverbs usually end in 'ly' - and in the phrases condition the additional information distracts from recognition of this pattern.

\section{Conclusions}

The results imply that user interfaces for syntactic search should show candidate relationships augmented with a list of phrases in which they occur. A list of phrases is the most recognizable presentation for clausal relationships ( $34 \%$ better than the baseline), and is as good as a list of words for the other types of relations, except adverb modifiers. For adverb modifiers, the list of words is the most 
recognizable presentation. This is likely because Enlglish adverbs usually end in '-ly' are therefore a distinctive set of words.

The list of candidates can be ordered by frequency of occurrence in the collection, or by an interestingness measure given the search word. As the user becomes more familiar with a given relation, it may be expedient to shorten the cues shown, and then re-introduce them if a relation has not been selected after some period of time has elapsed. If phrases are used, there is a tradeoff between recognizability and the space required to display the examples of usage. However, it is important to keep in mind that because the suggestions are populated with items from the collection itself, they are informative.

The best strategy, phrases, had an overall success rate of only $55 \%$, although the intended user base may have more familiarity with grammatical relations than the participants did, and therefore may perform better in practice. Nonetheless, there is room for improvement in scores, and it may be that additional visual cues, such as some kind of bracketing, will improve results. Furthermore, the current study did not test three-word relationships or more complex combinations of structures, and those may require improvements to the design.

\section{Acknowledgements}

We thank Björn Hartmann for his helpful comments. This work is supported by National Endowment for the Humanities grant HK-50011.

\section{References}

I Androutsopoulos, GD Ritchie, and P Thanisch. 1995. Natural language interfaces to databases-an introduction. Natural Language Engineering, 1(01):2981.

Peter Anick and Raj Gopal Kantamneni. 2008. A longitudinal study of real-time search assistance adoption. In Proceedings of the 31st annual international ACM SIGIR conference on Research and development in information retrieval, pages 701-702. ACM.

Aron Culotta and Andrew McCallum. 2005. Reducing labeling effort for structured prediction tasks. In $A A A I$, pages 746-751.

Marie-Catherine De Marneffe, Bill MacCartney, Christopher D Manning, et al. 2006. Generating typed dependency parses from phrase structure parses. In $L R E C$, volume 6, pages 449-454.
Fred Gibbs and Trevor Owens. 2012. Building better digital humanities tools. DH Quarterly, 6(2).

Lynette Hirschman, Alexander Yeh, Christian Blaschke, and Alfonso Valencia. 2005. Overview of biocreative: critical assessment of information extraction for biology. BMC bioinformatics, 6(Suppl 1):S1.

HV Jagadish, Adriane Chapman, Aaron Elkiss, Magesh Jayapandian, Yunyao Li, Arnab Nandi, and Cong Yu. 2007. Making database systems usable. In Proceedings of the 2007 ACM SIGMOD international conference on Management of data, pages 1324. ACM

Milos Jakubicek, Adam Kilgarriff, Diana McCarthy, and Pavel Rychlỳ. 2010. Fast syntactic searching in very large corpora for many languages. In PACLIC, volume 24, pages 741-747.

Stephan Kepser. 2003. Finite structure query: A tool for querying syntactically annotated corpora. In EACL, pages 179-186.

Aniket Kittur, Ed H Chi, and Bongwon Suh. 2008. Crowdsourcing user studies with mechanical turk. In Proceedings of the SIGCHI conference on human factors in computing systems, pages 453-456. ACM.

Catherine Lai and Steven Bird. 2010. Querying linguistic trees. Journal of Logic, Language and Information, 19(1):53-73.

Roger Levy and Galen Andrew. 2006. Tregex and tsurgeon: tools for querying and manipulating tree data structures. In LREC, pages 2231-2234.

Diana Lynn MacLean and Jeffrey Heer. 2013. Identifying medical terms in patient-authored text: a crowdsourcing-based approach. Journal of the American Medical Informatics Association.

Aditi Muralidharan and Marti A Hearst. 2013. Supporting exploratory text analysis in literature study. Literary and Linguistic Computing, 28(2):283-295.

William C Ogden and Susan R Brooks. 1983. Query languages for the casual user: Exploring the middle ground between formal and natural languages. In Proceedings of the SIGCHI conference on $\mathrm{Hu}$ man Factors in Computing Systems, pages 161-165. ACM.

Georg Rehm, Oliver Schonefeld, Andreas Witt, Erhard Hinrichs, and Marga Reis. 2009. Sustainability of annotated resources in linguistics: A web-platform for exploring, querying, and distributing linguistic corpora and other resources. Literary and Linguistic Computing, 24(2):193-210.

Philip Resnik, Aaron Elkiss, Ellen Lau, and Heather Taylor. 2005. The web in theoretical linguistics research: Two case studies using the linguists search engine. In Proc. 31st Mtg. Berkeley Linguistics Society, pages 265-276. 
Ben Shneiderman and Catherine Plaisant. 2010. Designing The User Interface: Strategies for Effective Human-Computer Interaction, 5/e (Fifth Edition). Addison Wesley.

Rion Snow, Brendan O'Connor, Daniel Jurafsky, and Andrew Y Ng. 2008. Cheap and fast-but is it good?: evaluating non-expert annotations for natural language tasks. In Proceedings of the conference on empirical methods in natural language processing, pages 254-263. Association for Computational Linguistics.

Jan-Philipp Soehn, Heike Zinsmeister, and Georg Rehm. 2008. Requirements of a user-friendly, general-purpose corpus query interface. Sustainability of Language Resources and Tools for Natural Language Processing, 6:27.

David Ward, Jim Hahn, and Kirsten Feist. 2012. Autocomplete as research tool: A study on providing search suggestions. Information Technology and $\mathrm{Li}$ braries, 31(4):6-19. 